\title{
BMJ Open Understanding the implementation of 'sick day guidance' to prevent acute kidney injury across a primary care setting in England: a qualitative evaluation
}

Anne-Marie Martindale, ${ }^{1}$ Rebecca Elvey, ${ }^{1}$ Susan J Howard, Sheila McCorkindale, ${ }^{2}$ Smeeta Sinha, ${ }^{3}$ Tom Blakeman $^{1}$

To cite: Martindale A-M, Elvey R, Howard SJ, et al. Understanding the implementation of 'sick day guidance' to prevent acute kidney injury across a primary care setting in England: a qualitative evaluation. BMJ Open 2017;7:e017241. doi:10.1136/ bmjopen-2017-017241

- Prepublication history for this paper is available online. To view these files please visit the journal online (http://dx.doi. org/10.1136/bmjopen-2017017241).

Received 18 April 2017 Revised 15 August 2017 Accepted 29 August 2017

\section{(D) CrossMark}

${ }^{1}$ National Institute for Health Research Collaboration for Leadership in Applied Health Research and Care, Salford Royal NHS Foundation Trust, Salford, UK

${ }^{2} \mathrm{NHS}$ Salford Clinical

Commissioning Group, Salford, UK

${ }^{3}$ Salford Royal NHS Foundation Trust, Salford, UK

Correspondence to Dr Anne-Marie Martindale; anne-marie.martindale@ manchester.ac.uk

\section{ABSTRACT}

Objectives The study sought to examine the implementation of sick day guidance cards designed to prevent acute kidney injury (AKI), in primary care settings.

Design Qualitative semistructured interviews were conducted and comparative analysis informed by normalisation process theory was undertaken to understand sense-making, implementation and appraisal of the cards and associated guidance.

Setting A single primary care health setting in the North of England.

Participants 29 participants took part in the qualitative evaluation: seven general practitioners, five practice nurses, five community pharmacists, four practice pharmacists, two administrators, one healthcare assistant and five patients.

Intervention The sick day guidance intervention was rolled out (2015-2016) in general practices $(n=48)$ and community pharmacies $(n=60)$. The materials consisted of a 'medicine sick day guidance' card, provided to patients who were taking the listed drugs. The card provided advice about medicines management during episodes of acute illness. An information leaflet was provided to healthcare practitioners and administrators suggesting how to use and give the cards.

Results Implementation of sick day guidance cards to prevent AKI entailed a new set of working practises across primary care. A tension existed between ensuring reach in administration of the cards to at risk populations while being confident to ensure patient understanding of their purpose and use. Communicating the concept of temporary cessation of medicines was a particular challenge and limited their administration to patient populations at higher risk of AKI, particularly those with less capacity to self-manage.

Conclusions Sick day guidance cards that focus solely on medicines management may be of limited patient benefit without adequate resourcing or if delivered as a standalone intervention. Development and evaluation of primary care interventions is urgently warranted to tackle the harm associated with AKI.

\section{INTRODUCTION}

Addressing the harm related to acute kidney injury (AKI) is a worldwide priority. ${ }^{1}$ AKI
Strengths and limitations of this study

- Using normalisation process theory has allowed important insights to emerge into the comprehension, use and appraisal of the acute kidney injury (AKI) sick day card initiative.

- Interviews with a range of professionals (general practitioners, nurses, community and practicebased pharmacists, a healthcare assistant, practice administrators) and patients enhanced understanding of the individual and collective working practises surrounding the professional implementation AKI sick day guidance cards.

- Patient recruitment to the qualitative evaluation via general practice was slow and yielded only five patient-participants. This limited the analysis of patient use of sick day guidance in everyday life.

- Future study design would benefit from greater alignment between quantitative and qualitative elements of an evaluation.

is characterised as a sudden reduction in kidney function over hours or days. ${ }^{2-4}$ It is a marker of illness severity and is seen as a 'force multiplier,' complicating episodes of acute illness. ${ }^{3}$ As a clinical syndrome, the majority of cases of AKI are due to a combination of underlying infection, hypovolaemia (low circulatory blood volume), hypotension (low blood pressure) and medication effects. ${ }^{3}$ Addressing these potentially modifiable factors are central to both the prevention and management of AKI and its associated burden. ${ }^{2-4}$

Across the UK, patient safety initiatives have been established to address the morbidity, mortality and costs linked to AKI. ${ }^{25-7}$ In Scotland, informed by findings from a primary care study conducted by NHS Highland, medicine sick day rules have been made 
available nationally through the Scottish Patient Safety Programme ${ }^{68}$ The introduction of medicine sick day rules relates to NHS Scotland Polypharmacy Guidance as well as national guidance, published by the National Institute for Health and Care Excellence (NICE) and by the Royal College of Physicians of Edinburgh UK. ${ }^{410}$ These publications highlight a need to consider temporary cessation of medicines at times of acute illness. ${ }^{4910}$ That is, during these episodes, 'any drug that reduces blood pressure, circulating volume or renal blood flow' increases the risk of AKI. ${ }^{3}$ Medicines that exacerbate this risk include non-steroidal anti-inflammatory drugs (NSAIDS), diuretics, ACE inhibitors and angiotensin II receptor blockers (ARBs). ${ }^{3}$ In addition, the Scottish medicine sick day rules refer to the temporary cessation of metformin, which may accumulate at times of reduced kidney function, resulting in an increased risk of adverse effects. ${ }^{6}$ The NHS Scotland 'Medicine Sick Day Rules' cards were developed through extraction of NHS Scotland Polypharmacy Guidance (2012) and were 'designed with input from pharmacists, doctors and patients'. ${ }^{10} 11$ They provide instructions on temporarily stopping these specific types of medicines during episodes of acute illness. ${ }^{68}$

In England, within NHS England's Patient Safety Domain, the Think Kidneys Programme (https:// www.thinkkidneys.nhs.uk) was established to tackle the harm associated with AKI. ${ }^{12}$ Through the programme, resources have been developed for primary and secondary care, including an Interim Position Statement on 'Sick Day' Guidance, which highlights a clinical equipoise surrounding the systematic implementation of sick day guidance. $^{13}$

It was in this wider context that a Clinical Commissioning Group (CCG), in partnership with the local hospital, embarked on service improvement initiatives to address the harm associated with AKI. Informed directly by the Scottish approach in conjunction with national guidance, ${ }^{468}$ the CCG sought to implement the use of sick day guidance across general practices and community pharmacies within its boundaries. The Sick Day Guidance Project including an overview of the organisation of primary healthcare in England is outlined in table 1 as well as figures 1A,B. In accordance with NHS England Think Kidneys guidance, the project entailed formal evaluation. With a view to providing a platform for future larger scale evaluation, the study sought to explore and understand processes underpinning the implementation of sick day guidance in primary care.

\section{METHODS}

\section{Study design}

Aligned with the project objectives, normalisation process theory (NPT) provided a sensitising framework to inform the topic guide and explore the context, administration, interpretation and use of sick day guidance cards across a single primary healthcare setting in England. ${ }^{14} 15$ NPT is a theory of implementation developed through an in-depth analysis of chronic illness care in general practice ${ }^{14}$ It is a sociological theory that provides a structure to explore the individual and group work that people do surrounding the implementation of a complex intervention. ${ }^{14-16}$

\section{Data sampling}

To explore the trajectory of implementation across the CCG, all general practices $(n=48)$, community pharmacies $(\mathrm{n}=60)$ and practice-based pharmacists $(n=4)$ involved in the project were invited to take part in the evaluation. Information packs were provided to explain what involvement entailed. To facilitate patient-participant engagement, general practices and community pharmacists were asked to provide information packs to patients who had received a card via a health practitioner. The final data sample of 29 interviews comprised: seven general practitioners (GPs), five practice nurses, five patients, five community pharmacists, four practice-based pharmacists, two managers (one medical practice manager and one community pharmacy manager) and a healthcare assistant, a person qualified to carry out routine healthcare tasks.

\section{Data collection}

Two qualitative researchers (A-MM; RE) conducted the 29 semistructured interviews. These were conducted with participants across the CCG between June 2015 and April 2016. Participants received an approved participant information sheet and consent form via post or email. Both were read by the researcher prior to interview and participants had the opportunity to ask questions and have them answered satisfactorily. Informed consent was gained before each interview. Interviews with the GPs, practice nurses, administrators and the healthcare assistant took place in private locations within their general practices. Interviews with community pharmacists were also held at private locations at their places of work. Interviews with patients occurred at their homes. Interviews with three of the practice-based pharmacists took place at their place of work; one took place on the phone. The two researchers did not know any of the participants prior to interview. The interviews ranged in length from 9 to 66 minutes (median=33 min). They were digitally audio-recorded in compliance with participants' consent and professionally transcribed.

Interview topic guides were developed to explore the work being undertaken by professionals and patients surrounding the use of sick day guidance cards. NPT was used to inform the areas of questioning. ${ }^{15}$ Topics for the health practitioners included previous knowledge of AKI and involvement in kidney health initiatives, their role in the intervention, sense-making and experiences of implementing and appraising the administration of sick day guidance cards. For patient-participant interviews, topics included: sensemaking around health and illness, the context of card 


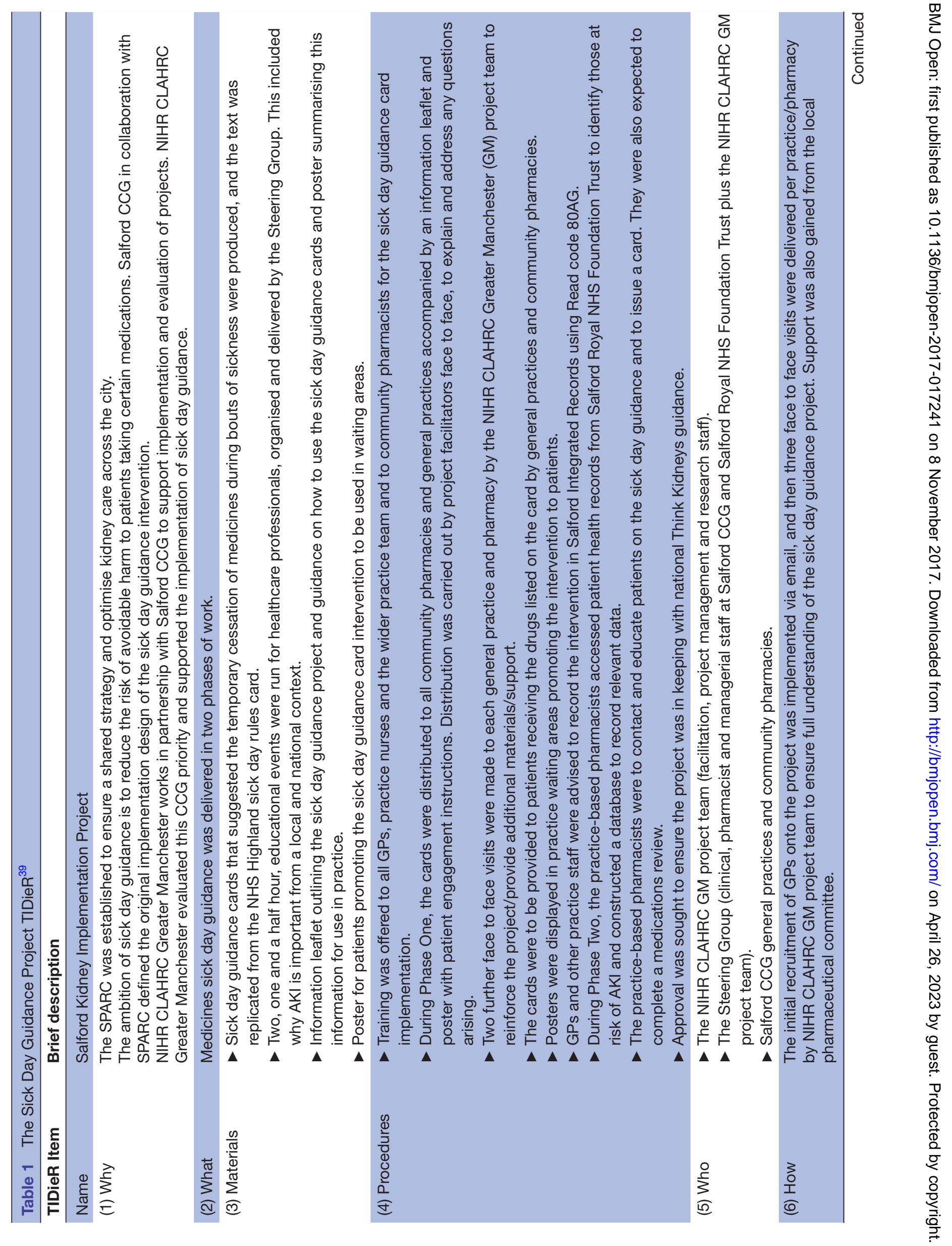




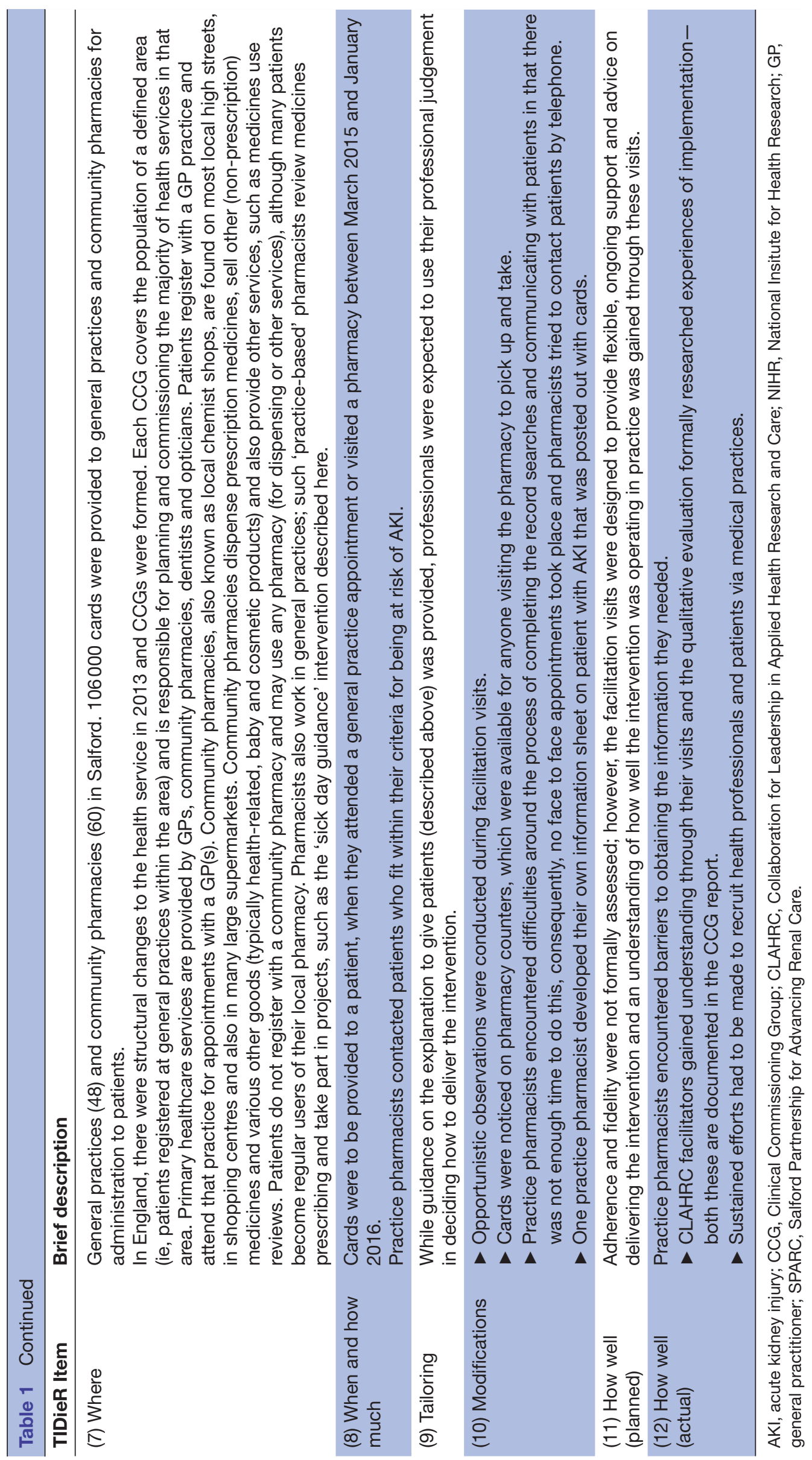




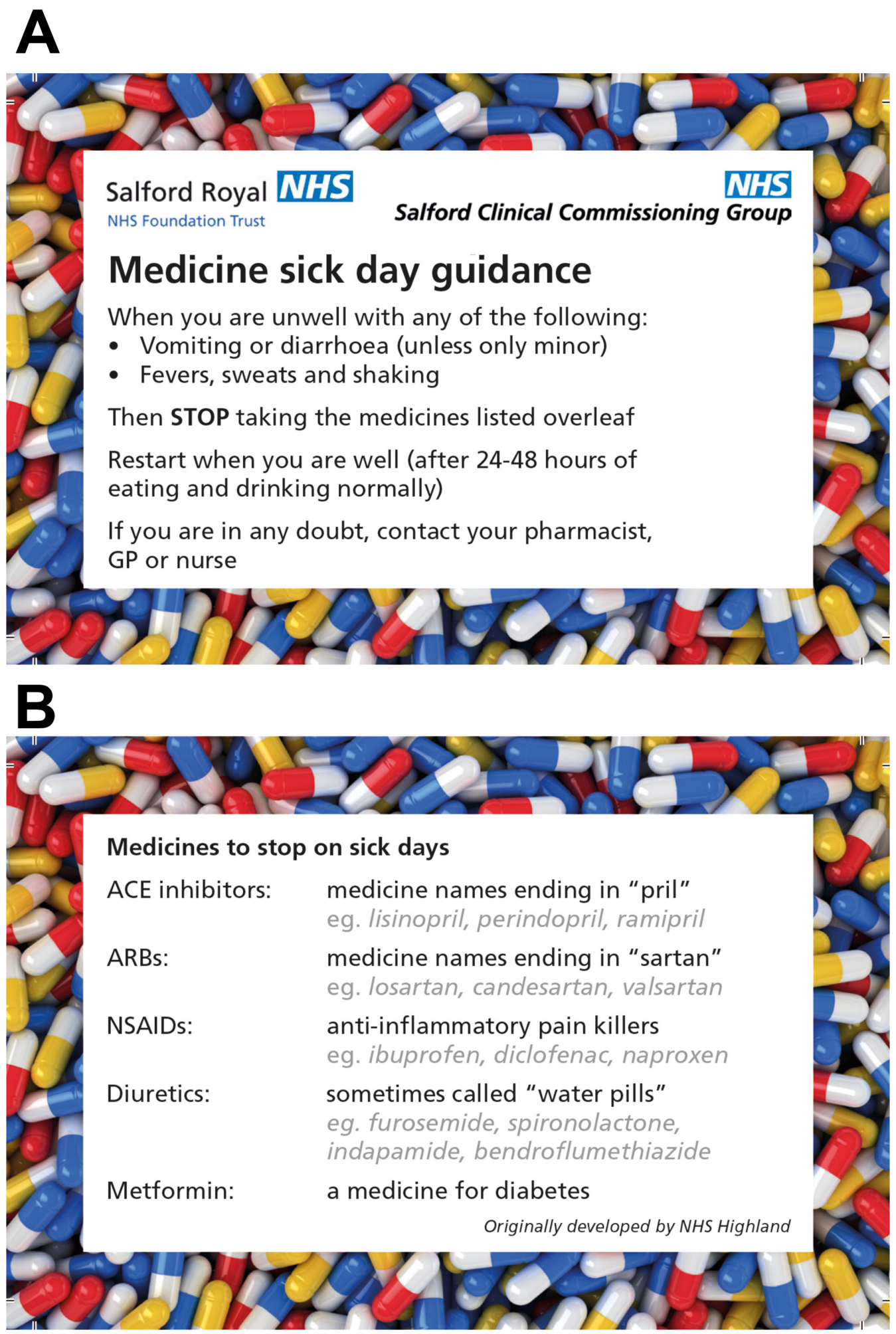

Figure 1 (A) and (B) Sick day guidance card used during this project. The NHS Highland sick day rules card was reproduced with new logos. ${ }^{68}$ ARB, angiotensin II receptor blockers; GP, general practitioner; NSAIDs, non-steroidal anti-inflammatory drugs.

giving and guidance explanation and comprehension and use of the guidance (table 2). Field notes about the encounter were written immediately after leaving the interview site and used to inform the analysis. Participants were asked if they wanted to receive a transcript post interview to check for accuracy, none did. 


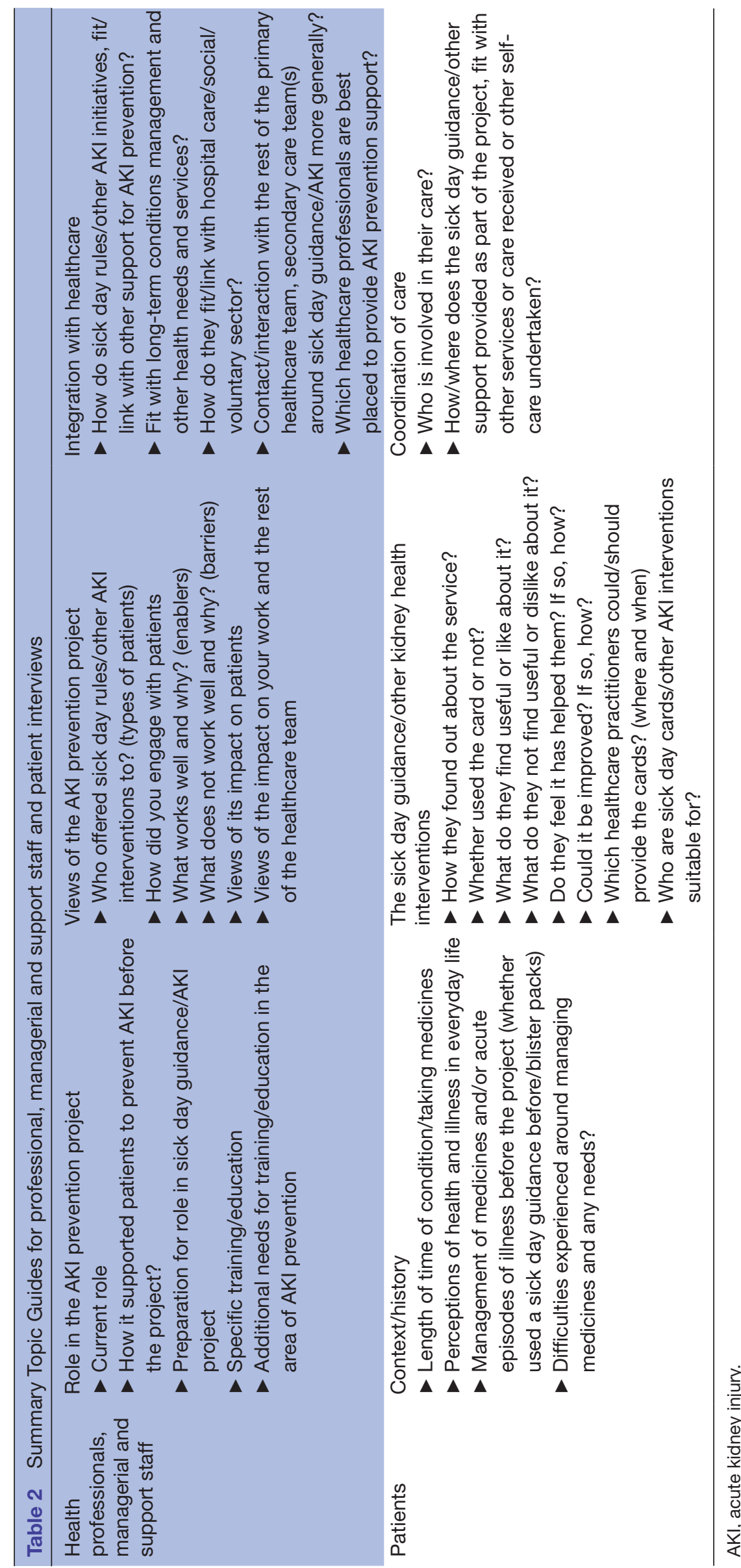

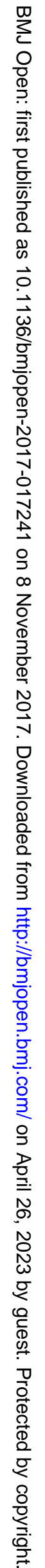




\section{Data analysis}

A-MM developed a thematic analysis framework using the evaluation objectives and the four core constructs of NPT to understand implementation. ${ }^{14}{ }^{15}$ NPT is concerned with social action rather than attitudes, and its four core constructs are coherence (sense-making), cognitive participation (relational work), collective action (operational work) and reflexive monitoring (appraisal). ${ }^{14} 15$ The NPT constructs provided a pragmatic structure to consider different types of work surrounding the implementation of sick day guidance cards. Furthermore, it provided a sensitising framework to explore the relationships between different types of work being undertaken. ${ }^{17}$ The questions asked of the health practitioner interview data included:

- how do they make sense of implementing the sick day card initiative? (coherence)

- what work have they done to implement the initiative? (operational work)

- how is the initiative being communicated or enacted by local others? (relational work)

- what judgments have been made about the initiative? (appraisal)

The questions we asked of the patient-participant data included:

- how does the participant make sense of health and illness? (coherence)

- what was the context of the participant receiving a card and guidance?

- how did they make sense of the card and implement the guidance in their day to day lives? (coherence, operational, relational work)

- how did they value the intervention? (appraisal).

As the interviews were completed and transcribed, data from each account were grouped according to role, which resulted in six datasets: GP, practice nurse and healthcare assistant, administration, community pharmacist, practice pharmacist and patient-participant. Thematic analysis using the transcripts, the audio recordings and the field notes was carried out by A-MM and TB. Each interview within a role group was analysed, and the findings were compared with those within the same group. Variations and similarities in context, sense-making, implementation and appraisal of the card were noted, explored and compared with the findings within and between role groups to enhance broader understanding. ${ }^{18}$ Key themes and tensions underpinning implementation emerged through comparative, contextual analysis of individual and collective working practises underpinning introduction of sick day guidance cards.

\section{RESULTS}

A version of the findings of this paper is included in a wider report that has been provided to the funding organisation. ${ }^{19}$ AKI was viewed as a new phenomenon and the implementation of sick day guidance cards entailed a new set of working practises. Analysis indicated that AKI prevention guidance was not necessarily a straightforward concept to understand or to communicate. Health practitioners thought the cards required some knowledge of illness symptoms and medicines and that patients had to decide how severe the symptoms were before acting or restarting their medication. One practice pharmacist stated:

'...patients don't understand what fever is... they think that if they've got a headache it's fever... we're trying to explain and they don't understand, or they say well, if I had a bout of diarrhoea do I stop the medication...it's severe. Well, what is severe, you know? Obviously it's very subjective...' (SKHIP13PP).

Comparative analysis highlighted a tension between the need to achieve reach to the populations deemed at risk (ie, those taking medicines specified on the card) and at the same time ensure comprehension concerning use of the guidance. There was evidence that this tension influenced the implementation of the sick day guidance intervention. The following sections describe the different approaches employed.

\section{Administration of the sick day guidance card in conjunction with face-to-face communication}

A common theme was health professionals and patients valuing the need to explain the guidance in person. One patient reflected:

'I don't think that it should be just put on a counter... I don't think, number one, they'll read it, number two, they'll digest what's on it, or number three, they'll apply it to themselves' (SKHIP22PA).

A practice nurse thought dialogue was also important to reduce miscommunication, avoid patient confusion and additional GP workload:

'I always explain ... There's no point giving someone a card if they don't understand what it's for... my grandma wouldn't understand that. She'd probably misinterpret that and...stop taking everything' (SKHIP25PN).

Analysis of health practitioner and patient accounts revealed that patients responded to the guidance in a variety of ways, not always as intended. One patient participant used the terms sickness and illness interchangeably and spoke of different classifications of illness. She asked which type the guidance card was referring to, to be confident of following the instructions properly:

'What do you define as illness...? Well, I suppose I don't know... I've got arthritis, that's not an illness it's just a thing of life when you get older... I've had spinal surgery, but they're not illnesses...' (SKHIP22PA).

Two health practitioners reported instances of patients with medication-associated diarrhoea stopping their tablets since receiving a card. This unintended consequence of the initiative lead to those patients being prescribed alternative medication to alleviate the side 
effect. A couple of patient-participant accounts revealed a lack of willingness to follow the guidance as it had not been implemented by their hospital specialist, whose opinion they trusted, and they did not want to make their condition worse:

\section{'I'd rather feel sick than have a problem with the high blood pressure...' (SKHIP31PA).}

The concept of temporary cessation of medicines required careful consideration, for example when to stop, restart and what dosage to reinstate:

'We don't have enough data or... best practice... if you stop the metformin or whatever medication how long do you stop

it for...? Then after a week are you going to restart them again on the ten milligram or are you going to start them on the 1.5, the 2.5...?' (SKHIP14GP).

Although valued by the health practitioners interviewed, implementation of sick day guidance initiative demanded extra work. In general practice, this was deemed less problematic when it fitted into existing longterm condition review appointments, particularly with practice nurses or healthcare assistants. In community pharmacies, implementation sat more readily within faceto-face medication review appointments or opportunistic over-the-counter interactions, including the purchase of NSAIDS such as ibuprofen. One community pharmacist used the purchase of antidiarrhoeal or sickness medications as an opportunity to administer AKI guidance:

'... when people have been coming in to buy stuff for sickness or diarrhoea... If it turns out that they're on one of the medications that's on the card, then we'll give them a card then as well and explain about it' (SKHIP5CP).

There were limits to the implementation of sick day guidance in patient populations deemed at increased risk of AKI. Concerns were expressed across the health professionals interviewed that the cards and temporary cessation of medications were not suitable for patients with cognitive impairments such as Alzheimer's disease, reduced literacy in English, those with advanced learning difficulties or visual impairments or for elderly housebound patients taking multiple medicines. One community pharmacist commented on the difficulties facing patients and carers using dosette box (blister pack) systems:

'they (patients) might have four or five tiny little white ones, and then if they're elderly or they can't see the markings, they don't know what tablet they should be stopping.... if it was a family member looking out for it, that would be I guess possible, but a lot of the carers are not allowed to alter any medication' (SKHIP7CP).

\section{Administration of sick day guidance cards to patients in conjunction with telephone consultations}

Phase Two of the project entailed Practice Pharmacists supporting the implementation of the sick day guidance cards in general practices (see table 1). All of the four CCG employed pharmacists valued and engaged with the project. However, they outlined difficulties fitting the implementation in with their pre-existing workload. There were more patients to work with than anticipated, and the searches, writing to patients, communicating with them and feeding the results back to GPs took longer to complete than the pharmacists described having time for.

To implement the project in this context, a decision was made to have telephone conversations with patients rather than face-to-face interactions. However, this created additional challenges. The phone calls took as long as the face-to-face encounters as the pharmacists expressed a professional need to do things 'properly'. They reported patients not always being happy to talk with a perceived stranger on the phone about their health. Patient understanding was harder to assess and patients did not necessarily agree to enact the guidance if they became ill. Unlike the face-to-face GP and practice nurse consultations, patients on the other end of the phone had no prior trusting relationship with the practice pharmacist. One pharmacist tried to mitigate some of these issues by talking with a GP in advance of phoning:

'...I'm not going to just pick up the phone and ring this patient now, I'm going to ask the GP what he thinks... for the slightly elderly- some patients, perhaps mental health issues... They obviously know their patients much better than I do so I always take their advice' (SKHIP11PP).

The community pharmacists also spoke of the difficulties of assessing patient comprehension in this way:

'I've had to phone patients ... if you've got a query or the prescription will be changed or we'll want to question something ... sometimes they're on the ball, they completely know, and sometimes they're just so confused'(SKHIP7CP).

Sick day guidance cards being administered without verbal or written communication

Instructions administered to health practitioners (figure 2) stressed the need for dialogue with patients to check understanding. However, accounts indicated that this did not always occur. Reasons included other work demands during a practice-based consultation, limited time for dialogue, forgetting to discuss it and some lack of confidence about what to say, partly because of the limited evidence base and so as not to confuse patients, especially those who were less fluent in English:

'we have quite a lot of different ethnicities here... they've got limited English I think they're not quite sure and it takes quite a while explaining ... about what medicines to stop, when to stop it, when to restart it...' (SKHIP10PN).

Though the community pharmacists were willing to talk with patients about the guidance cards, time shortages and other work demands impinged on implementation. One community pharmacist stated: 


\section{MEDICINES AND DEHYDRATION: SICK DAY GUIDANCE}

Offer the following information at the time of giving the card

- Some medicines shouldn't be taken when you have an illness that makes you dehydrated. This is because they can either increase the risk of dehydration or because dehydration can lead to potentially serious side effects of the medicine.

- The medicine you are taking that falls into this category is [tell patient which medicine].

- IIInesses that can cause dehydration are vomiting, diarrhoea and fever.

- This advice does not apply to minor sickness or diarrhoea, which means a single episode.

- If your medicines are in a blister pack you must take it to the chemists so the chemist can show you which ones you need to stop.

- If you have heart failure you may stop these medicines for a maximum of 48 hours but after that you need to contact your GP or heart failure team for further advice.

The list of medicines on the card is not exhaustive but they are highlighted because:

- diuretics can cause dehydration or make dehydration more likely in an ill patient;

- ACE inhibitors, angiotensin II receptor blockers and NSAIDs may impair kidney function in a dehydrated patient, which could lead to kidney failure;

- metformin dehydration increases the risk of lactic acidosis, a serious and potentially lifethreatening side effect of metformin.

Figure 2 Guidance provided to health practitioners (shortened form). GP, general practitioner; NSAIDs, non-steroidal antiinflammatory drugs.

'Half the time it's remembering to do it because you're thinking about that many different things' (SKHIP5CP).

In addition, they did not always have face-to-face contact with patients:

'we've got like 900 of our own patients and we just make the packs and then send them out and delivery, so we don't actually have that much patient contact' (SKHIP7CP).

Some health practitioners felt that the cards were self-explanatory. One practice nurse said:

'vomiting is vomiting and diarrhoea is diarrhoea' (SKHIP25PN).

However, others did not agree. One GP thought it was really important to provide patients with written material to aid understanding and compliance:

'with certain other sort of medicine regimes, we ask them to stop temporarily if there's a drug interaction and patients are okay with that, as long as you give them sort of written instructions and they know exactly why they're stopping. A lot of it is to do with the understanding. They don't like stopping things if they don't understand why...' (SKHIP20GP).

A couple of patient accounts referred to finding cards in public information areas of medical practices and community pharmacies. One patient who found a card in this manner wanted to share the sick day guidance message:

'...I went into the pharmacy last week, they were on the counter...I picked one up and brought it home ...I think it's such a good idea that I've given one to my sister' (SKHIP22PA).

Communication of AKI risk, but limited use of a sick day guidance card

One GP worked exclusively with patients in care homes across the CCG, which included patients who were diagnosed with cognition limiting conditions such as dementia. Though the guidance messages were deemed pertinent to these groups of patients more vulnerable to AKI, their use was limited due to a potential lack of understanding:

'So we have the card. We didn't use it a lot... We used it to give to the carers. I used it to give to a few of the patients that have capacity' (SKHIP14GP).

The need for appropriate training for carers, nursing staff and associated social workers was raised, beyond the level of the sick day guidance card. Specifically there was felt to be an ongoing need for health practitioners to highlight the importance of fluid management in conjunction with medicines management: 
'...they (dementia patients) ended up not eating or drinking, worsening of the renal function and become unwell and they end up in hospital...' So it's working with the carer as well to understand.... It's serious things that they might die from, not being hydrated' (SKHIP14GP).

\section{DISCUSSION}

\section{Principal findings}

Implementation of sick day guidance cards to prevent community based AKI entailed a new set of working practises. The temporary cessation of medicines during episodes of acute illness was not necessarily a straightforward concept to understand or communicate. Comparative analysis of participants' accounts highlighted a tension between ensuring reach in administration of the cards to at risk populations while being confident to ensure patient understanding of their purpose and use.

\section{Strengths and weaknesses of this study}

Unlike an earlier study, ${ }^{20}$ a key strength of this evaluation was to conduct an in-depth exploration of systematic rollout across a single healthcare setting. The study was hypothesis generating, and use of NPT provided a sensitising framework for data collection and analysis. ${ }^{14-16}$ Recognising that all theories have the potential to structure and constrain analysis, NPT was chosen as it ensured that a range of individual and collective working practises were considered during analysis. ${ }^{14}{ }^{15}$ Methods to enhance the trustworthiness of the findings, including their transferability, entailed exploring types of work undertaken in both general practices and community pharmacies as well as their use by a range of health professionals in these different settings. ${ }^{21}$

The study entailed comparative analysis of both patient and professional accounts in order to explore their use in clinical interactions as well as in everyday life. Thematic analysis has illuminated a key tension between achieving reach while ensuring comprehension of the card and its instructions. However, a larger sample size might have resulted in the identification of additional themes that may have had an impact on this theoretical framework. Further research is required to enhance patient understanding and use. Professional accounts allowed descriptions of experiences of use by patients, though difficulties were encountered recruiting patient-participants who had experiences of having used a sick day guidance card at times of acute illness. It is important to acknowledge that only five patients were interviewed in spite of extensive recruitment efforts. It is not possible to determine how many patients received information packs as we did not ask practices to keep a record, to reduce work load. Health professionals did not always pass on the evaluation recruitment packs to patients, and the patients we interviewed had not used the cards to date, which could help to explain limited patient involvement. Workload pressures were cited as reasons for health professionals declining to participate in the evaluation.

During the course of the interviews, health practitioners were asked about patient sense-making, use and appraisal of the guidance cards. In light of limited patient involvement, these accounts became more important. We acknowledge that they are third order interpretations; our interpretations of what health practitioners reported about patients' sense-making, appraisal and use of the cards. However, the comparative approach taken has facilitated understanding of the pluralistic journeys of the cards and their intended and unintended messages and trajectories from card giver to patient across the 29 interviews. Future studies may benefit from sampling patients who have been coded in general practice as having been provided sick day guidance (ie, Read Code 8OAG. 'Provision of information about AKI ${ }^{22}$ and also who have been coded with an episode of acute illness (eg, gastroenteritis, acute respiratory infection). In doing so, this this would enable purposeful sampling according to medical history including evidence of multimorbidity. As stated in the CCG report, 106000 cards (see table 1) were distributed across general practices and community pharmacies within the time frame of the project. ${ }^{19}$ However, community pharmacists were not required to record administration to patients and inaccuracies in coding in general practice limited the potential for a robust quantitative analysis. Future study design would benefit from greater alignment between quantitative and qualitative elements of an evaluation. ${ }^{19}$

\section{Comparison with other studies}

In terms of professional responsibility, there are recognised boundaries to the role of GPs in supporting self-management. ${ }^{23}$ The findings of this study resonate and build on the results of previous research, which highlighted issues around the consistency of clinical message and the additional work required to reduce the risk of harm from AKI using medicines management interventions. ${ }^{2024}$ The intervention was conducted at a time when concern was raised that UK general practice workload may be at 'saturation point. ${ }^{, 25}$ Results suggested that this influenced engagement with the CCG-led initiative.

Though currently available through the Scottish Patient Safety Programme ${ }^{6}$ the findings from this qualitative study resonate with recently published literature, which highlights a need for a more robust evidence base surrounding both the implementation and effectiveness of sick day guidance cards. ${ }^{26-28}$ A recent systematic review showed that 'there is no evidence of the impact of drug cessation interventions on AKI incidence during intercurrent illness in primary or secondary care. ${ }^{.26}{ }^{28}$ In terms of implementation, studies evaluating AKI interventions in secondary care indicate that establishing clinician approval is critical with a need for intervention design to take into account 'how technologies, people and organisations dynamically interact' in order for AKI interventions to become integrated into routine clinical practice. ${ }^{29}{ }^{30}$ Interventions that disrupt workflow 'may not 
be sustainable even if there has been a positive impact on care. $^{29}$

Results from a population-based cohort study indicate that patient comorbidities including chronic kidney disease are much more strongly associated with AKI and that treatment with either an ACE Inhibitor or an ARB is only associated with a small increase in AKI risk. ${ }^{27}$ That is, younger patients with limited comorbidity (eg, on ACEI for treatment of hypertension) have a low absolute risk of AKI, while patients living with multimorbidity in whom there may be professional concerns about ensuring effective risk communication, have a much higher risk of AKI. ${ }^{27}$

\section{Implications for clinicians, policy makers and future research}

In the UK, NICE recommends raising awareness of AKI in higher risk population groups with specific reference to patients who: have existing CKD; have had a previous episode of illness complicated by AKI and/or have neurological or cognitive impairment and who may be reliant on carers for support with fluid intake during an acute illness (eg, those with cognitive impairment). ${ }^{31}$ This may help address a knowledge gap in patient and public understanding of the importance in the maintenance of kidney health. A survey conducted in 2014 on behalf of NHS England indicated that 'about half of the population in Great Britain do not think their kidneys make urine' and 'only an eighth (12\%) of interviewees thought their kidneys had a role in processing medicines. ${ }^{32}$ However, the findings from this study suggest an evidence base is urgently warranted to determine how best to resource effective self-management support for higher risk patient populations. Targeting patients who have had an episode of illness complicated by AKI may be particularly important. As a marker of vulnerability, data from a Welsh study showed that around $50 \%$ of their patient population died within 14 months; the study also revealed high rates of hospital readmission. ${ }^{33}$ Of the 733 patients discharged following a hospital admission complicated by AKI, there were 498 rehospitalisation events in a 6-month period. $^{33}$

The NHS England Urgent and Emergency Care Review also emphasised the need for better support for people to self-care. ${ }^{34}$ Our analysis in conjunction with the research by Mansfield et al ${ }^{27}$ suggests sick day guidance cards alone, that focus solely on temporary cessation of medicines, are unlikely to be sufficient to reduce the harm associated with AKI. The CCG chose to implement the Scottish (NHS Highland) Medicine Sick Day Rules card without significant modification of content or format. ${ }^{6}$ However, the current intervention may need modifying, to make it suitable for use with various populations, such as provision in languages other than English. For example, recognising the risks of the 'triple whammy' combination of NSAIDS prescribed in conjunction with diuretics and renin-angiotensin system inhibitors (ie, ACE inhibitors and ARBs), is there potential for misunderstanding if NSAIDS are included in a sick day guidance card administered to patients with heart failure ${ }^{35}$ Both usability testing and experience-based codesign are methodological approaches that may optimise the development of an intervention that takes into account patient and carer experience. ${ }^{36}$ The findings suggest other strategies may need to be resourced to prevent AKI in people with complex health and social care needs such as those living with dementia. A key issue raised was to provide better education and support for carers (both professional and informal). The Royal College of General Practitioners has provided guidance on the development of 'carer friendly' practises and the establishment of Patient Participation Groups may be a mechanism to resource and integrate support for carers into the organisation of acute care. ${ }^{3738}$

\section{CONCLUSION}

The findings from this qualitative evaluation suggest that there are boundaries to the implementation of sick day guidance cards to prevent acute kidney injury in primary care. A common theme was the need to ensure patient understanding of their purpose and use. Communicating the concept of temporary cessation of medicines was a particular challenge and limited their administration to patient populations at higher risk of AKI, particularly those with less capacity to self-manage. The analysis suggests that sick day guidance cards that focus solely on medicines management may be of limited benefit without either adequate resourcing or if delivered as a standalone intervention. Development and evaluation of a primary care intervention encompassing a range of initiatives to tackle the harm associated with AKI is warranted.

Acknowledgements The authors would like to thank the project steering group for their input and guidance throughout the study.

Contributors TB, RE, SJH, SM and SS contributed to the conception and design of the work. RE and A-MM collected the data, RE, A-MM and TB analysed the data. A-MM, TB, RE and SJH drafted the manuscript and all authors contributed to interpreting the data, revising the work for intellectual content, agree to be accountable for the work and have approved the manuscript.

Funding This project was funded by the National Institute for Health Research Collaboration for Leadership in Applied Health Research and Care (NIHR CLAHRC) Greater Manchester and NHS Salford CCG. The NIHR CLAHRC Greater Manchester is a partnership between providers and commissioners from the NHS, industry and the third sector as well as clinical and research staff from the University of Manchester.

Disclaimer The views expressed in this article are those of the authors and not necessarily those of the NHS, NIHR or the Department of Health.

\section{Competing interests None declared.}

Ethics approval Ethical approval was gained from Leeds West Research Ethics Committee (REC Reference Number: 15/YH/0174). Informed consent was gained from all participants prior to interview.

Provenance and peer review Not commissioned; externally peer reviewed.

Data sharing statement The data have been stored securely with password protected files to ensure confidentiality, in keeping with the research protocol and good data management guidelines. It will not be shared.

Open Access This is an Open Access article distributed in accordance with the terms of the Creative Commons Attribution (CC BY 4.0) license, which permits others to distribute, remix, adapt and build upon this work, for commercial use, provided the original work is properly cited. See: http://creativecommons.org/ licenses/by/4.0/ 
(c) Article author(s) (or their employer(s) unless otherwise stated in the text of the article) 2017. All rights reserved. No commercial use is permitted unless otherwise expressly granted.

\section{REFERENCES}

1. Mehta RL, Cerdá J, Burdmann EA, et al. International Society of Nephrology's 0by25 initiative for acute kidney injury (zero preventable deaths by 2025): a human rights case for nephrology. Lancet 2015;385:2616-43.

2. Kidney Disease Improving Global Outcomes (KDIGO) Acute Kidney Injury Work Group. KDIGO Clinical Practice Guideline for Acute Kidney Injury. Kidney International Supplement, 2012;2:1-138.

3. NHS England, UK Renal Registry, Think Kidneys. Acute Kidney Injury Best Practice Guidance: Responding to AKI Warning Stage Test Results for Adults in Primary Care. Think Kidneys, 2016.

4. National Institute for Health and Care Excellence. Acute Kidney Injury: prevention, detection and management (CG169). London: NICE, 2013.

5. NHS England, UK Renal Registry, Think Kidneys. Acute Kidney Injury Warning Alert Best Practice document. England: Think Kidneys, 2014.

6. NHS Scotland, Health Improvement Scotland, Scottish Patient Safety Programme. Medicine Sick Day Rules Cards. NHS Scotland, Health Improvement Scotland, 2016. http://www. scottishpatientsafetyprogramme.scot.nhs.uk/programmes/primarycare/medicine-sick-day-rules-card (accessed 4 Apr 2017).

7. Kerr M, Bedford M, Matthews B, et al. The economic impact of acute kidney injury in England. Nephrol Dial Transplant 2014;29:1362-8.

8. Morrison C, Wilson M. Medicine Sick Day Rules Cards Intermin Evaluation. NHS Highland, 2014. http://www.knowledge.scot.nhs. uk/media/CLT/ResourceUploads/4055542/NHSH\%20interim\% 20evaluation\%20medicine\%20sick\%20day\%20rules.pdf (accessed 7 Jan 2015).

9. Feehally J, Gilmore I, Barasi S, et al. RCPE UK consensus conference statement: Management of acute kidney injury: the role of fluids, e-alerts and biomarkers. J R Coll Physicians Edinb 2013;43:37-8.

10. Scottish Government. Polypharmacy guidance. NHS Scotland, 2015 http://www.sehd.scot.nhs.uk/publications/DC20150415polypha rmacy.pdf (accessed 2 Apr 2015).

11. Morrison C, Wilson M. Medicine Sick Day Rules cards: a safe and effective tool to improve medicines safety in NHS Highland. Int J Pharm 2015;23:S2. //onlinelibrary.wiley.com/doi/10.1111/ijpp.12213/ pdf

12. NHS England, UK Renal Registry [internet], Think Kidneys. http:// www.thinkkidneys.nhs.uk/ (accessed 7 Apr 2015).

13. NHS England, UK Renal Registry, Think Kidneys. "Sick day" guidance in patients at risk of Acute Kidney Injury: an Interim Position Statement from the Think Kidneys Board. Think Kidneys, 2015.

14. May CR, Mair F, Finch T, et al. Development of a theory of implementation and integration: Normalization Process Theory. Implement Sci 2009;4:29.

15. Murray E, Treweek S, Pope C, et al. Normalisation process theory: a framework for developing, evaluating and implementing complex interventions. BMC Med 2010;8:63.

16 Normalization Process Theory. Toolkit and NoMAD instrument. http:// www.normalizationprocess.org (accessed 10 Oct 2015).

17 Tsang JY, Blakeman T, Hegarty J, et al. Understanding the implementation of interventions to improve the management of chronic kidney disease in primary care: a rapid realist review. Implement Sci 2016;11:47.

18 Silverman S. Interpreting Qualitative Data: Methods for Analysing Talk, Text and Interaction. London: Thousand Oaks, 2001.

19 NIHR Collaboration for Leadership in Applied Health Research and Care Greater Manchester. Salford Clinical Commissioning Group Sick Day Guidance Project: Final Report. Manchester: NIHR CLAHRC
GM, 2016 http://clahrc-gm.nihr.ac.uk/our-work/kidney-health/salfordsick-day-guidance/

20 Morris RL, Ashcroft D, Phipps D, et al. Preventing Acute Kidney Injury: a qualitative study exploring 'sick day rules' implementation in primary care. BMC Fam Pract 2016;17:91.

21. Lincoln Y, Guba EG. Naturalistic Inquiry. London: Sage, 1985.

22. NHS North West Commissiong Support Unit. Specification for Read V2 coding for: Salford Clinical Commissioning Group. Salford Standards Read Code Dictionary. NHS North West Commissioning Support Unit, 2016.

23. Blakeman T, Macdonald W, Bower P, et al. A qualitative study of GPs' attitudes to self-management of chronic disease. Br J Gen Pract 2006;56:407-14.

24. Phipps DL, Morris RL, Blakeman T, et al. What is involved in medicines management across care boundaries? A qualitative study of healthcare practitioners' experiences in the case of acute kidney injury. BMJ Open 2017;7:e011765.

25. Hobbs FDR, Bankhead C, Mukhtar T, et al. Clinical workload in UK primary care: a retrospective analysis of 100 million consultations in England, 2007-14. The Lancet 2016;387:2323-30.

26. Morden A, Horwood J, Whiting P, et al. The risks and benefits of patients temporarily discontinuing medications in the event of an intercurrent illness: a systematic review protocol. Syst Rev 2015;4:1-6.

27. Mansfield KE, Nitsch D, Smeeth L, et al. Prescription of reninangiotensin system blockers and risk of acute kidney injury: a population-based cohort study. BMJ Open 2016;6:1-6.

28. Whiting $P$, Morden A, Tomlinson LA, et al. What are the risks and benefits of temporarily discontinuing medications to prevent acute kidney injury? A systematic review and meta-analysis. BMJ Open 2017;7:e012674.

29. Kanagasundaram NS, Bevan MT, Sims AJ, et al. Computerized clinical decision support for the early recognition and management of acute kidney injury: a qualitative evaluation of end-user experience. Clin Kidney J 2016;9:57-62.

30. Oh J, Bia JR, Ubaid-Ullah M, et al. Provider acceptance of an automated electronic alert for acute kidney injury. Clin Kidney $J$ 2016:9:567-71.

31. National Institue for Health and Care Excellence. Acute kidney injury Quality. London: NICE, 2014. org.uk/guidance/qs76

32. NHS England, UK Renal Registry, Think Kidneys. Findings from Ipsos MORI survey - July 2014. Understanding what the public know about their kidneys and what they do. Think Kidneys, 2014

33. Wonnacott A, Meran S, Amphlett B, et al. Epidemiology and outcomes in community-acquired versus hospital-acquired AKI. Clin J Am Soc Nephrol 2014;9:1007-14.

34. England NHS. Leeds: Urgent and Emergency Care Review Team. High quality care for all, now and for future generations: Transforming urgent and emergency care services in England, Urgent and Emergency Care Review. NHS England, 2013.

35. Dreischulte T, Morales DR, Bell S, et al. Combined use of nonsteroidal anti-inflammatory drugs with diuretics and/or reninangiotensin system inhibitors in the community increases the risk of acute kidney injury. Kidney Int 2015;88:396-403.

36. Locock L, Robert G, Boaz A, et al. Testing accelerated experiencebased co-design: a qualitative study of using a national archive of patient experience narrative interviews to promote rapid patientcentred service improvement. Southampton (UK): NIHR Journals Library, 2014. https://www.ncbi.nlm.nih.gov/books/NBK259580/

37. Royal College of General Practitioners. RCGP Supporting Carers in General Practice:Summary report on GP practice journeys towards improved carer identification and support. London: Royal College of General Practitioners, 2013.

38. NHS Employers. 2015/16 General Medical Services (GMS) contract: Guidance for GMScontract 2015/16. London: NHS Employers, 2015.

39. Hoffmann TC, Glasziou PP, Boutron I, et al. Better reporting of interventions: template for intervention description and replication (TIDieR) checklist and guide. BMJ 2014;348:g1687. 